\title{
Promoting Antimicrobial Stewardship and Combating Antimicrobial Resistance: Redefining the role of Critical Care Physicians
}

\author{
Kaniz Fatema
}

Antimicrobial resistance is a global threat and it has very recently received global attention. When the critically ill patients of Intensive Care Unit (ICU) are infected with antibiotic resistant organisms, it adversely affects their outcome. The key driver of antibiotic resistance is inappropriate antibiotic usage. Studies have shown that $30 \%$ to $60 \%$ of antibiotics prescribed in ICUs are unnecessary, inappropriate or suboptimal. ${ }^{1,2}$

It has been observed that "Antimicrobial Stewardship Program" (ASP) also known as Antibiotic Stewardship Program, help the clinicians improve the infection cure rate, reduce treatment failure and significantly reduce antibiotic resistance. ${ }^{3}$ Infectious Diseases Society of America (IDSA), Society for Healthcare Epidemiology of America (SHEA) and Pediatric Infectious Diseases Society (PIDS) defined antibiotic stewardship as "coordinated interventions designed to improve and measure the appropriate use of [antibiotic] agents by promoting the selection of the optimal [antibiotic] drug regimen including dosing, duration of therapy, and route of administration". ${ }^{4}$

The high antibiotic resistance observed in hospital patient population especially those of ICU, increases their mortality and morbidity, length of stay in hospital. It also increases length of hospital stay, cost of care and contributes to emergence and dissemination of microbial resistant strains. To combat this life-threatening problem, ASP must be implemented in ICUs. Centers for Disease Control and Prevention (CDC), IDSA, SHEA and PIDS suggested implementation ofASP in all acute-care hospitals, and ICUs, both adult and pediatric. ${ }^{4} \mathrm{ASP}$ for critically ill patients encompasses the following strategies.

Timely administration of appropriate antibiotic is a major outcome determinant for ICU patients with severe sepsis and septic shock. As the critically ill patients may present with features SIRS due to non-infectious causes, it is important to identify patients with true infections. Besides history and physical findings, biological markers like C-reactive protein (CRP), soluble-triggering receptor expressed on myeloid cells-I, or procalcitonin (PCT) may guide the intensivists to recognize true bacterial infections and facilitate therapeutic decisions. Rapid viral tests should be used (if available) for respiratory pathogens to reduce use of antibiotic in viral pneumonia. ${ }^{4}$.With the same aim, non-culture-based fungal markers should be used to optimize anti-fungal.

Pattern of microbes and their resistance pattern vary from one country to another, one hospital to another, even among ICUs in the same hospital. So, physicians involved in the

management of the critically ill patients should have knowledge of local bacteriological epidemiology along with the current guidelines. Institutional antibiograms are helpful for development of guidelines for empiric therapy. It is better to stratify antibiogram by age of the patients, infection site, co-morbidities, or acquisition of infection in community vs healthcare setting for better formulation of empiric treatment. ${ }^{4}$

Knowledge of pharmacokinetic-pharmacodynamics (PK-PD) characteristics for optimal use of antibiotics is important. Critically ill patients of ICU have either hyper dynamic circulation, or altered fluid balance due to $3^{\text {rd }}$ spacing or altered protein, or renal failure, or hepatic dysfunction, or on organ support systems like renal replacement therapy, or extra-corporeal membrane oxygenation (ECMO). All these effect the PK of antibiotics. ${ }^{5}$ It is better to give antibiotics in continuous or extended infusions in ICU patients. ${ }^{6}$ Antibiotic doses should be adjusted based on pathogen MICs and daily free antibiotic concentration. Optimized PK-PD targets in ICU patients reduces clinical failure rates and prevents adverse events due to too high (toxic) drug levels. ${ }^{6}$

Because of non-specific clinical signs of infection, patients with non-infectious causes may be treated with antibiotics in ICUs. So, as soon as the culture and other supportive results (eg chest X-ray, PCT) are available, unnecessary antibiotics must be withdrawn. Therapy can be de-escalated when culture reports do not yield significant number of organisms specific for that specimen, and do not show growth of resistant organisms. If the isolates are sensitive to narrow spectrum antibiotics, empiric broad spectrum antibiotics can be replaced in hemodynamically stable patients. Serial PCT measurement is another tool to monitor the response and de-escalation of antibiotics

Prolonged therapy with antibiotics in community acquired infection (CAI) and health-care associated infections (HAI) facilitates colonization with antibiotic-resistant bacteria preceding recurrent infections. So, based on the therapeutic responses and inflammatory markers, reduction of treatment duration of antibiotics is possible even in patients with severe sepsis or septic shock. This strategy helps in containing health-care costs and limit emergence of bacterial resistance. However it may not work in immunosuppressed patients, in patients whose course deteriorates despite treatment and in those infected with multi-drug resistant organism.

A successful ASP requires an interdisciplinary team composed of prescribers of antibiotics (i.e., clinicians/intensivists), clinical pharmacists with infectious disease training, microbiologists, hospital epidemiologist, 
laboratory staff, information technology staff and nurses. ${ }^{7}$ Formal training in infectious diseases and antibiotic stewardship benefits the program. Prior authorization for some antibiotics by the ASP team or hospital authority, and prospective audit with feedback on prescriptions are also essential components of ASP. ASP must be accompanied by a comprehensive infection prevention and control program that includes hand hygiene, standard and transmission based precautions, and cleaning and disinfection

Implementation of ASP has posed as a challenge for critical care physicians of Bangladesh. Several studies done in Bangladesh have shown high level of antibiotic resistance in ICU patients ${ }^{8-10}$. Unfortunately there is neither any formulary policy in hospitals here, nor any prescription guideline for physicians. Any pharmacy or drug store inside or outside hospitals may dispense drug without prescription. We have no national drug policy regarding sale of drug specially antibiotics. There is no nationwide data or statistics on consumption of higher antibiotics which are mostly abused by registered as well as quake physicians. Moreover, pharmaceutical industries manufacturing antibiotics entice physicians for profit to prescribe broad spectrum antibiotics without clear guidelines. Critical care physicians have a leadership role to play to disseminate ASP as they have a much bigger stake in caring for critically ill patients. Our Govt. should strictly regulate licensing drug stores/ pharmacies and enforce prescription policies. Bangladesh Medical Association (BMA), the biggest body representing all physicians of the country should make this its most important agenda by sending a memorandum of national action plan on implementation of ASP to our ministry of health.

There have been alarming headlines in Newspapers all over the world that very few broad spectrum antibiotics to treat sepsis, are in the pipe line (because of paucity of funding for research) in the coming decade. So ASP is our only hope to save millions of lives of critically ill patients suffering from sepsis.

\section{References:}

1. Bergmans DC, Bonten MJ, Gaillard CA et al. Indications for antibiotic use in ICU patients: a one-year prospective surveillance. J Antimicrob Chemother 1997; 39: 527-35.

2. Kollef MH. Optimizing antibiotic therapy in the intensive care unit setting. Crit Care 2001; 5: 189-95.

3. Malani AN, Richards PG, Kapila S, Otto MH, Czerwinski J, Singal B. Clinical and economic outcomes from a community hospital's antimicrobial stewardship program. American journal of infection control 2013;41(2):145-148.

4. Barlam TF, Cosgrove SE, Abbo LM et al. Implementing an antibiotic stewardship program: Guidelines by the Infectious Diseases Society of America and the Society for Healthcare Epidemiology of America. Clin Infect Dis 2016; doi: 10.1093/cid/ciw118

5. Luyt CE, Brechot N, Trouillet JL, Chastre J. Antibiotic stewardship in the intensive care unit. Crit Care 2014; 18:480.

6. Lodise TP, Drusano GL. Pharmacokinetics and pharmacodynamics: optimal antimicrobial therapy in the intensive care unit. Crit Care Clin 2011; 27: 1-18.

7. https://www.cdc.gov/getsmart/healthcare/implementation/coreelements.html

8. Fatema K, Ahsan ASMA, Barai L, et al. Bacterial profile and their antibiotic resistance in an ICU of Bangladesh: comparison of four studies from 2004 to 2011. Bangladesh Crit Care J 2016; 3 (2): 79-85.

9. Shahunja KM, Salam MA, Ahmed T, et al. Bacterial isolates from tracheal aspirates and their antimicrobial susceptibility in mechanically ventilated children with pneumonia to an urban critical care ward.Bangladesh Crit Care J 20124; 2(2): 60-4.

10. Mannan MA, Kashem MA, Mohammed FR, Rabbani R, Islam MM. Microbiological profile of severe lower respiratory tract infection in intensive care unit of a tertiary care center of Dhaka, Bangladesh. Bangladesh Crit Care J 20124; 2(2): 53-6.
Dr. Kaniz Fatema

FCPS (Medicine), MD (Critical Care Medicine)

Assistant Professor

Department of Critical Care Medicine

BIRDEM General Hospital

Dhaka 1000, Bangladesh

Email: drkanizfatemasb@gmail.com 